\title{
Hexagonal optical patterns in anisotropic non-linear media
}

\author{
A. V. Mamaev $\left(^{*}\right)$ and M. Saffman \\ Department of Optics and Fluid Dynamics, Risø National Laboratory \\ Postbox 49, DK-4000 Roskilde, Denmark
}

(received 17 November 1995; accepted in final form 7 May 1996)

PACS. 42.60Jf - Beam characteristics: profile, intensity, and power; spatial pattern formation and dynamics.

PACS. 42.65Sf - Dynamics of nonlinear optical systems; optical instabilities, optical chaos, and optical spatio-temporal dynamics.

PACS. $42.65 \mathrm{Hw}$ - Phase conjugation, optical mixing, and photorefractive effect.

\begin{abstract}
We present experimental observations of hexagonal patterns in strongly anisotropic non-linear optical media. Close to threshold rolls are observed. As the non-linearity is increased a transition from rolls to hexagons, leading finally to a pure hexagonal state, is observed. A mean-field model, in agreement with experiments, shows that the anisotropy locks the far-field orientation of the hexagons, while leaving their rotational symmetry undisturbed.
\end{abstract}

Hexagonal spatial patterns have been observed in many different areas of the natural sciences [1], [2]. These include the Rayleigh-Bénard experiment in fluids, chemical reactions, and non-linear optics. Pattern formation phenomena that were originally observed in other fields have been reproduced in recent years in non-linear optics. Current interest in nonlinear optics goes, however, deeper than demonstrating the universality of pattern formation. The widespread use of optical technologies in information transmission and processing points towards applications of optical patterns [3]. At a more fundamental level there are aspects of the physics of non-linear optics that are not found in other fields. In the work described here we report on the formation of hexagonal spatial patterns in a photorefractive crystal with strongly anisotropic non-linear coupling. In our experiment the non-linear coupling is zero along one axis of the observed pattern. The anisotropy breaks rotational symmetry in the plane of the pattern, and clamps the orientation of the participating wave vectors. Nonetheless, we observe rotationally symmetric hexagonal patterns, identical to those seen in isotropic media.

Transverse spatial patterns are observed when two beams counterpropagate in a non-linear optical medium. Both beams are modulated by the perturbation to the dielectric permittivity caused by each beam, which leads to transverse modulational instability [4]-[6]. The nonlinear stage of this instability leads to regular transverse patterns most often with hexagonal symmetry [6], [7]. Patterns have been observed in a wide variety of optical materials, including atomic vapours [7], liquid crystals [8], photorefractives [9], and organic films [10].

In a medium that is characterized by a Kerr-type third-order non-linearity the coupling is isotropic, for interacting beams with parallel linear polarization. In photorefractive crystals the non-linearity is in general strongly anisotropic. This is due to the structure of the electro-optic and dielectric tensors, and to the influence of any externally applied field. Despite this, it

$\left({ }^{*}\right)$ Permanent address: Institute for Problems in Mechanics, Russian Academy of Sciences, Prospekt Vernadskogo 101, Moscow, 117526 Russia.

(c) Les Editions de Physique 


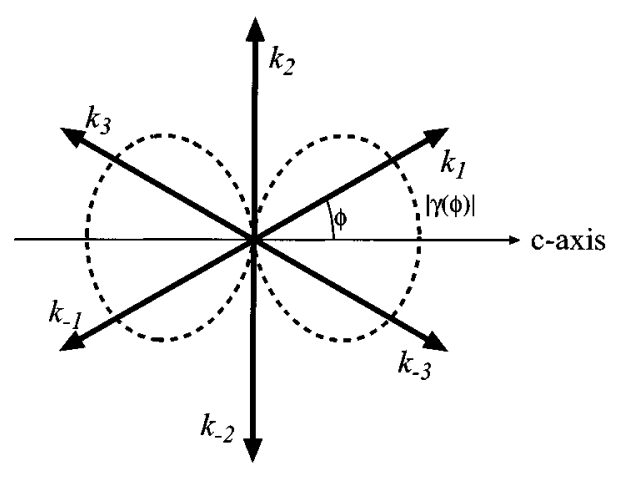

Fig. 1.

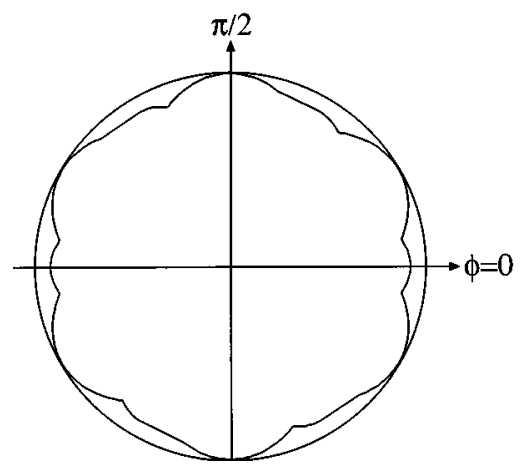

Fig. 2.

Fig. 1. - Orientation of wave vectors and anisotropic coupling diagram. The angular dependence of $|\gamma(\phi)|$ was calculated using $\epsilon_{1} / \epsilon_{3}=0.53$, which corresponds to SBN:60.

Fig. 2. - Intensity $\max \left(\left|f_{i}\right|^{2}\right)$ of the stationary hexagonal solution of eq. (3) as a function of the orientation angle $\phi$ for $\alpha=0.73$, which is slightly beyond the threshold for instability of the roll solution. The circle represents the case of isotropic coupling.

is possible to find configurations where the coupling is almost perfectly isotropic. In the geometry first demonstrated by Honda [9] the interacting beams propagate along the crystalline symmetry axis, and interact due to the formation of reflection gratings. The deviation from isotropy is small, being proportional to $\theta_{\mathrm{h}}^{2}$, where $\theta_{\mathrm{h}}(\sim 0.01$ in photorefractive experiments) is the far-field half-cone angle of the hexagons.

In the experiments reported here the non-linearity is anisotropic. The beams propagate perpendicular to the crystal c-axis, and interact via the formation of transmission gratings. This leads to the coupling diagram shown in fig. 1, where the dashed curve shows the magnitude of the non-linear coupling $\gamma$ as a function of the grating rotation angle $\phi$. In photorefractive media $\gamma$ is a complex quantity. As shown in [11], the instability threshold is minimized for $\gamma$ real. In photorefractive media with predominantly diffusive charge transport the refractive index grating is shifted with respect to the interference pattern and $\gamma$ is almost purely imaginary. This leads to very high instability thresholds. To make $\gamma$ real we apply an external electric field along the c-axis which leads to drift-driven charge transport, and unshifted gratings [12]. In the limit of $\theta_{\mathrm{h}} \ll 1$ the real part of $\gamma$ increases linearly with the external field. Grating vectors rotated by an angle $\phi$ thus see an effective coupling strength proportional to $r_{\text {eff }}(\phi) E_{\text {ext }}(\phi) /\left(\epsilon_{3} \cos ^{2}(\phi)+\epsilon_{1} \sin ^{2}(\phi)\right)$, where $r_{\text {eff }}$ is the effective electro-optic coefficient, $E_{\text {ext }}$ is the externally applied field, and $\epsilon$ is the dc dielectric tensor. In the experimental geometry described below, using SBN:60 as the non-linear medium, we have $\gamma(\phi)=\gamma_{0} \cos ^{2}(\phi) /\left(\cos ^{2}(\phi)+\frac{\epsilon_{1}}{\epsilon_{3}} \sin ^{2}(\phi)\right)$, where the relevant material parameters are lumped into the constant $\gamma_{0}$. The non-linear coupling for gratings oriented perpendicular to the c-axis is identically zero.

To see how hexagonal patterns arise in this situation we write the forward and backward propagating beams in the form

$$
\begin{aligned}
& F\left(\mathbf{r}_{\perp}, z, t\right)=F_{0} \exp [i k z]\left[1+\sum_{j=1}^{3}\left(f_{j} \exp \left[i \vartheta_{j}\right]+\text { c.c. }\right)\right] \\
& B\left(\mathbf{r}_{\perp}, z, t\right)=B_{0} \exp [-i k z]\left[1+\sum_{j=1}^{3}\left(b_{j} \exp \left[i \vartheta_{j}\right]+\text { c.c. }\right)\right]
\end{aligned}
$$


where $\vartheta_{j}=\mathbf{k}_{j} \cdot \mathbf{r}_{\perp}$, and $k$ is the wave number in the medium. We assume implicitly that the transverse wave vectors lie on a hexagon and satisfy $\left|\mathbf{k}_{j}\right|=k_{\mathrm{h}}=k \theta_{\mathrm{h}}$. Equations (1) are then substituted into the paraxial wave equation, retaining phase-matched terms up to third order in the perturbations. The amplitude of the non-linear contribution to the refractive index due to the coupling of perturbations $f_{i}, f_{j}$ takes the form $\delta n_{i j}=\gamma\left(\phi_{i j}\right) f_{i} f_{j}^{*} /\left(\left|f_{i}\right|^{2}+\left|f_{j}\right|^{2}+\right.$ $I_{\text {sat }} /\left|F_{0}\right|^{2}$ ), where $I_{\text {sat }}$ is the effective saturation intensity. To first order in the perturbations there is no contribution from the intensity term in the denominator and the resulting linear equations for photorefractive media are analogous, except for effects due to anisotropy, to those for Kerr media [5, 11]. At higher order there are contributions from the expansion of the denominator and the structure of the photorefractive equations is more complicated than those for Kerr media. In the present experiments the non-linearity is only weakly saturated $\left(I_{\text {sat }}>\left|F_{0}\right|^{2},\left|B_{0}\right|^{2}\right)$ and we will neglect such additional terms. The equations for the perturbations then take the form

$$
\begin{aligned}
& \frac{\mathrm{d} f_{1}}{\mathrm{~d} z}+i \frac{k_{\mathrm{h}}^{2}}{2 k} f_{1}=i \gamma_{1} \frac{2}{1+q}\left[f_{1}+q b_{1}\right]+i \frac{2}{1+q}\left[\gamma_{2}\left(f_{2}^{*}+q b_{2}^{*}\right) f_{3}^{*}+\gamma_{3}\left(f_{3}^{*}+q b_{3}^{*}\right) f_{2}^{*}\right]+ \\
& +\frac{i}{1+q}\left[\gamma_{1}\left(\left|f_{1}\right|^{2} f_{1}+q\left(b_{1} f_{1}^{*}\right) b_{1}\right)+2 \gamma_{2}\left(\left|f_{3}\right|^{2} f_{1}+q\left(b_{3} f_{3}^{*}\right) b_{1}\right)+2 \gamma_{3}\left(\left|f_{2}\right|^{2} f_{1}+q\left(f_{2} b_{2}^{*}\right) b_{1}\right)\right]+ \\
& +i \frac{2}{1+q}\left[\gamma_{12}\left(\left|f_{2}\right|^{2} f_{1}+q\left(b_{2} f_{2}^{*}\right) b_{1}\right)+\gamma_{13}\left(\left|f_{3}\right|^{2} f_{1}+q\left(f_{3} b_{3}^{*}\right) b_{1}\right)\right]
\end{aligned}
$$

where the remaining equations are found by permutation of the indices and $q=\left|B_{0}\right|^{2} /\left|F_{0}\right|^{2}$. The coupling constants are defined by $\gamma_{i}=\gamma(\phi+(i-1) \pi / 3)$ (where $\phi$ is as shown in fig. 1), $\gamma_{12}=\gamma(\phi+\pi / 6), \gamma_{23}=\gamma(\phi+\pi / 2)$, and $\gamma_{13}=\gamma(\phi+5 \pi / 6)$. Equations (2) should be solved with $f_{i}, b_{i}$ equal to zero at the ends of the non-linear medium. Very close to threshold only the first term on the right-hand side of (2) is significant and we find a roll instability [4], [5] with $\phi=0$ which maximizes $\gamma_{1}$.

In isotropic media rolls are unstable close to threshold, and a hexagonal state is seen [13]. This is not the case here (see frame "350" in fig. 4), where rolls are in fact stable close to threshold. To see this qualitatively, we consider the mean-field analogues of eqs. (2) [14], [15]. Putting $f_{i}=b_{i}$, which is appropriate for focusing media [15], and $q=1$ for simplicity, we get equations for the temporal evolution of the mean amplitudes of the form

$$
\frac{\mathrm{d} f_{i}}{\mathrm{~d} t}=2\left(\gamma_{i}+\Gamma_{0}\right) f_{i}+2\left(\gamma_{j}+\gamma_{k}\right) f_{j}^{*} f_{k}^{*}-\left[\gamma_{i}\left|f_{i}\right|^{2}+2\left(\gamma_{k}+\gamma_{i j}\right)\left|f_{j}\right|^{2}+2\left(\gamma_{j}+\gamma_{i k}\right)\left|f_{k}\right|^{2}\right] f_{i},
$$

where $\{i, j, k\}=1,2,3$, and $\Gamma_{0}$ is an effective longitudinal growth rate. For isotropic media $\gamma_{i}=\gamma_{i j}=\gamma$ and (3) reduces to the generic amplitude equations that describe roll-hexagon competition [13]. The amplitude of the stationary roll solution close to threshold is $\left|f_{10}\right|^{2}=$ $2\left(\gamma_{1}+\Gamma_{0}\right) / \gamma_{1}\left(\gamma_{1} \equiv \gamma(0)\right)$, so $\Gamma_{0} \sim-\alpha \gamma_{i}$, where $\alpha$ is a positive constant less than 1 , gives a small amplitude solution. Linear analysis shows that the roll solution aligned along $\phi=0$ is stable for $\gamma_{2}-\alpha \gamma_{1}-2\left[(1-\alpha)\left(\gamma_{2}+\gamma_{12}+\sqrt{2(1-\alpha)}\left(\gamma_{1}+\gamma_{2}\right)\right]<0\right.$. Given the angular dependence of the coupling in SBN this condition is satisfied for $\alpha>0.74$, or $\left|f_{10}\right|^{2}<0.52$.

For larger non-linearity the roll solution is unstable and we observe, eventually, a fully developed hexagonal structure. The hexagon is symmetric although the anisotropy fixes the angular orientation. To find the stationary orientation we assume the $f_{i}$ real with equal amplitudes and obtain a quadratic equation for $f_{i}$ from (3). As seen in fig. 2 the pattern has maximal amplitude when one of the pattern wave vectors is oriented at $\phi=\pi / 2$. This is reasonable since this orientation minimizes the strength of the linear coupling that gives rolls. As the non-linearity is increased (smaller values of $\alpha$ ) the angular selectivity decreases. 


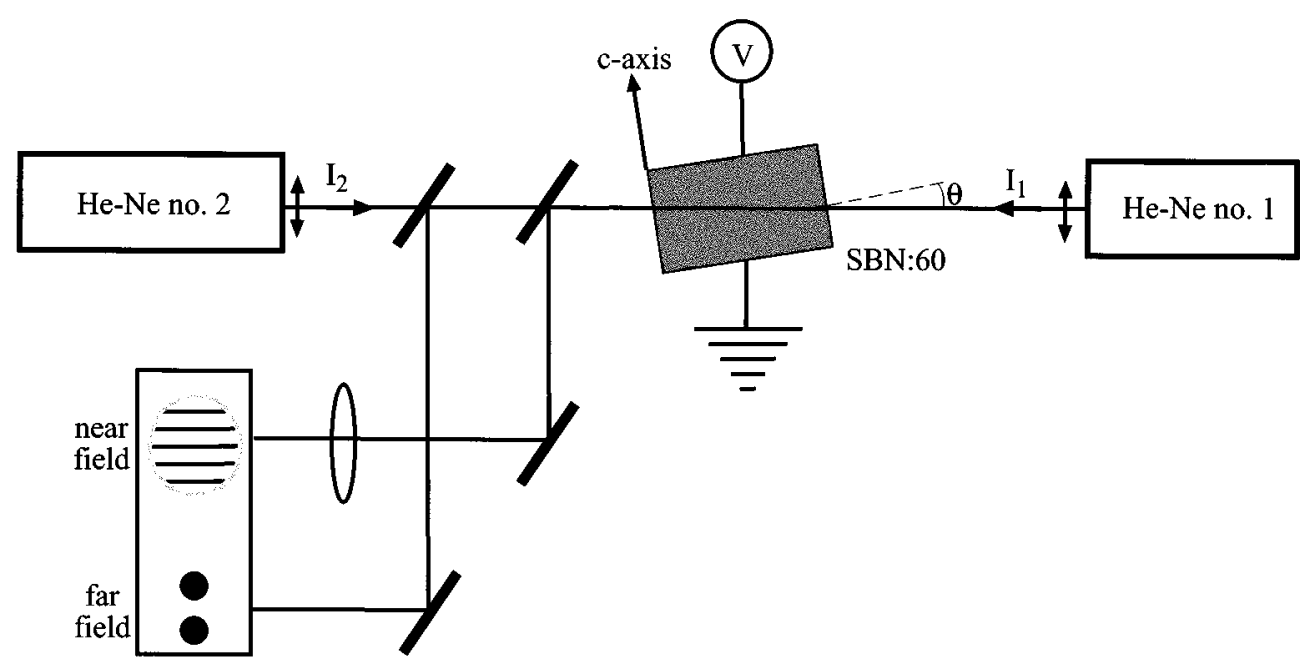

Fig. 3. - Experimental set-up.

The experimental geometry is shown in fig. 3. The beams from two HeNe lasers with $\lambda=632.8 \mathrm{~nm}$ and diameters of about $1.5 \mathrm{~mm}$ were arranged to counterpropagate in a photorefractive crystal of SBN:60, lightly doped with $0.002 \%$ by weight Ce. The possibility of forming reflection gratings was excluded due to the structure of the electro-optic tensor, and the fact that the beams were derived from separate lasers. The beams propagated in the horizontal plane roughly perpendicular to the crystal c-axis, and were polarized in the horizontal plane along the c-axis. The crystal measured $10 \mathrm{~mm}$ along the direction of propagation, and was $9 \mathrm{~mm}$ wide along the c-axis. The crystal was tilted by a small angle in the horizontal plane $\theta=9.7$ degrees in order to avoid interference from internal reflections. The beams incident on the crystal had powers of $I_{1}=4.5 \mathrm{~mW}$ and $I_{2}=12.5 \mathrm{~mW}$. The near- and far-field distributions of beam 1 after passage through the crystal were projected onto a screen and recorded with a CCD camera.

A variable dc voltage was applied along the c-axis to control the value of non-linear coupling. In addition, the crystal was illuminated from above with an incoherent white-light source. The incoherent illumination serves two purposes. First, it raises the background conductivity to ensure steady-state drift-dominated charge transport, and unshifted refractive index gratings. Second, the strong stimulated scattering (fanning) that normally occurs when mm-sized beams propagate through SBN is reduced, since the incoherent illumination also reduces the nonlinearity. By choosing an appropriate level of illumination it is possible to observe transverse instability while reducing the interference due to fanning. The incoherent illumination had an effective intensity of $I_{\text {incoh }}=1.4\left(I_{1}+I_{2}\right)$. The experiments were thus performed in a regime where the non-linearity is only weakly saturated. The residual fanning propagated at a much larger angle than that characterizing the pattern formation, and is not visible in the figures below.

Figure 4 shows the observed patterns for different values of applied voltage. The direction of the c-axis is horizontal in the figures. At zero voltage the non-linearity is small and there is no visible instability. The threshold of visible instability occurs at several hundred volts, which is not inconsistent with the analysis of ref. [11]. At $V=350$ volts a roll pattern with several defects is observed. Note that in the corresponding far-field picture the excitation is tilted slightly away from the c-axis. By repeating the experiment at different locations in the crystal, we found that the initial tilt direction varies from position to position. The instability 

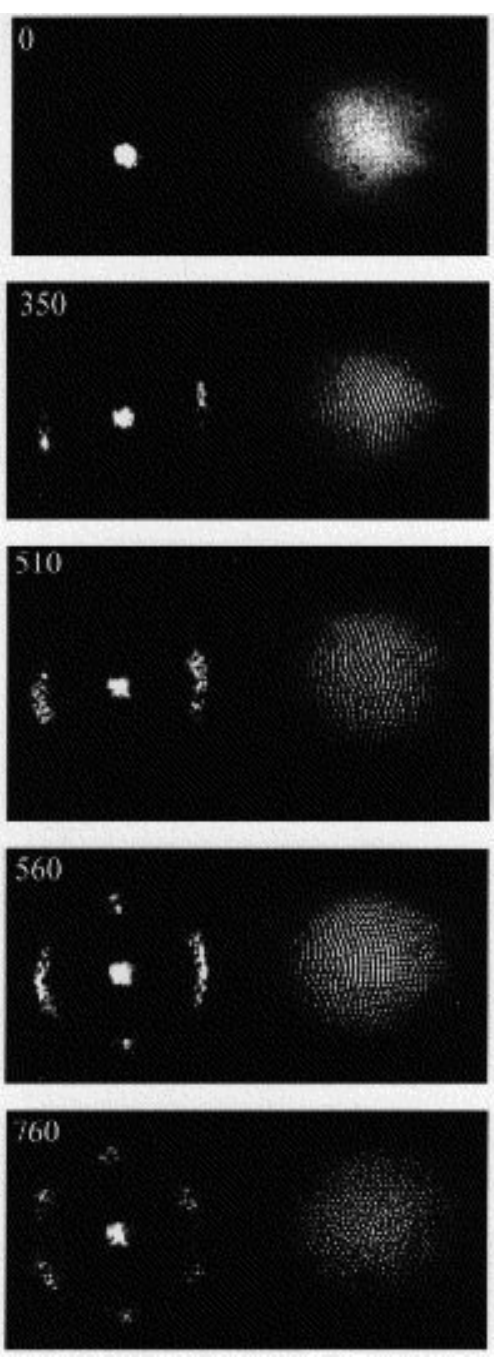

Fig. 4. - Near- and far-field patterns for different applied voltages. The c-axis is horizontal in the figures.

is seeded by surface and internal scattering in the medium. Since the magnitude and direction of the seeding varies with location, the instability orientation close to threshold can also vary.

At 510 volts the rolls begin to give way gradually to a two-dimensional tiling. The farfield pattern shows a pair of arc segments lying across the c-axis, in addition to very weak components perpendicular to the c-axis. At 560 volts roll, square, and hexagonal symmetries coexist. Inspection of the near-field pattern shows that the dominant symmetry varies with position. This may be attributed to the Gaussian-type intensity envelope of the beams leading to position-dependent non-linearity. At 760 Volts the near-field pattern has a fully developed hexagonal symmetry. The near-field pattern is characterized by a large number of defects which results in the six far-field spots having a speckled structure. The orientation of the far-field spots was fixed, in agreement with the orientation shown in fig. 2 and we did not observe the rotation seen using an isotropic reflection grating geometry [9]. It should be emphasized that the final hexagonal pattern has one set of wave vectors oriented approximately parallel with 
the direction of zero non-linearity. The same far-field patterns, with different near-field details, were observed on beam 2 .

Inspection of the far-field pattern yields no measureable indication of distortion of the hexagonal structure. The far-field full-cone angle was measured to be 1.47 degrees, which is close to the theoretical estimate [4] of $2 \sqrt{n \lambda / l_{\text {crystal }}}=1.40$ degrees, which is strictly valid only for $q=1$. The polarity of the applied voltage was chosen to give a self-focusing non-linearity. Changing the sign of the voltage gives a defocusing non-linearity for which we observed no evidence of pattern formation. This is in agreement with theoretical analyses of a transmission grating interaction which show no defocusing instability at finite transverse wave vector [11], [15]. In order to emphasize that the pattern formation is due to a counterpropagation instability, we propagated a single beam through the crystal with $V=760$ volts. The resulting picture was indistinguishable from frame " 0 " of fig. 4. For higher voltages the propagation of even a single beam becomes non-linear and we observe large-scale filamentation of the beam $[16]$.

In summary, we have analysed hexagonal patterns in self-focusing media with a strongly anisotropic non-linearity. Close to threshold rolls oriented along the direction of largest nonlinearity, are stable. At higher non-linearities a symmetric hexagonal pattern, locked with one wave vector perpendicular to the direction of largest non-linearity, is stable. Experimental observations are in good agreement with a mean-field analysis that accounts for the anisotropy.

This work was supported by a grant from the Danish Natural Science Research Council.

\section{REFERENCES}

[1] Newell A. C., Passot T. and Lega J., Annu. Rev. Fluid Mech., 25 (1993) 399.

[2] Cross M. C. and Hohenberg P. C., Rev. Mod. Phys., 65 (1993) 851.

[3] Vorontsov M. A. and Miller W. B. (Editors), Self-Organization in Optical Systems and Applications in Information Technology (Springer, Berlin) 1995.

[4] Vlasov S. N. and Talanov V. I., in Optical Phase Conjugation in Nonlinear Media, edited by V. I. Bespalov (Institute of Applied Physics, USSR Academy of Sciences, Gorki) 1979.

[5] Firth W. J. and Paré C., Opt. Lett., 13 (1988) 1096.

[6] Grynberg G., Opt. Commun., 66 (1988) 321.

[7] Grynberg G., Le Bihan E., Verkerk P., Simoneau P., Leite J. R. R., Bloch D., Le Boiteux S. and Ducloy M., Opt. Commun., 67 (1988) 363.

[8] Macdonald R. and Eichler H. J., Opt. Commun., 89 (1992) 289.

[9] Honda T., Opt. Lett., 18 (1993) 598.

[10] Glückstad J. and Saffman M., Opt. Lett., 20 (1995) 551.

[11] Saffman M., Montgomery D., Zozulya A. A., Kuroda K. and Anderson D. Z., Phys. Rev. A, 48 (1993) 3209.

[12] Günter P., Phys. Rep., 93 (1982) 199.

[13] Ciliberto S., Coullet P., Lega J., Pampaloni E. and Perez-Garcia C., Phys. Rev. Lett., 65 (1990) 2370.

[14] Courtois J. Y. and Grynberg G., Opt. Commun., 87 (1992) 186.

[15] Geddes J. B., Indik R. A., Moloney J. V. and Firth W. J., Phys. Rev. A, 50 (1994) 3471.

[16] Mamaev A. V., Saffman M., Anderson D. Z. and Zozulya A. A., to be published in Phys. Rev. A (1996). 